\title{
The effect of using supplementing on some physiological variables and slowing the appearance of fatigue in volleyball players
}

\section{Prof. Dr/ Youssef Dahab Ali}

\section{Prof. Dr/ Alaa El-Din Mohamed Eliwa}

\section{Doctoral researcher/ Mahmoud Mustafa Essa}

\section{Research Summary:}

Athletes are continuously searching for means to raise their performance level to the extent that exceeds their individual capabilities to achieve sportive achievements and reach advanced positions and at all levels, as increasing training loads and its dosages no longer fulfill the aspirations of athletes_;one of the most important nutritional supplements that have major impact on building the body and providing the necessary needs in rationing its construction is the Arginine compound, which is an amino acid that helps the body in building protein. Arginine has emerged in recent years as a nutritional supplement that enhances energy sources and increases the athlete's ability to endure performance. It directly affects the central nervous system (CNS) and various metabolic processes and the general result is to maintain glycogen due to increasing fat oxidation and reducing carbohydrate oxidation.

The study aims to identify the effect of using a nutritional supplement on some physiological variables and to slow the appearance of fatigue for volleyball 1 players.

The researcher used the experimental method for one group via using the pre and post measurement design as this is appropriate to the nature of this research.

Among the most important results are the following, Eating Arginine Acid as a nutritional supplement before the effort of volleyball players teams has led to an improvement in the disposal of lactic acid and a decrease in triglycerides in the body. Arginine intake helps in the function of Coenzyme and Enzyme to produce energy and to perform continuously, as well as in the urea cycle inside the body as a catalyst, and in the lactic acid cycle to get rid 
of harmful waste in the body. Eating Arginine Acid as a nutritional supplement before the effort of volleyball players has led to improve physiological functional responses that are affected by exercise, as the samples begin to take the nutritional supplement 15 minutes.

\section{Opening words:}

a nutritional supplement- physiological variables - fatigue

\section{Introduction and research problem:}

Arginine has emerged in recent years as a nutritional supplement that enhances energy sources and increases the athlete's ability to endure performance. It directly affects the central nervous system (CNS) and various metabolic processes and the general result is to maintain glycogen due to increasing fat oxidation and reducing carbohydrate oxidation, so it is important that coaches and specialists search for what helps the athlete to raise their endurance via improving the mechanisms of energy production, maintaining supplies in muscles and raising the level of nervous impulse via its use. The efficiency of preparing athletes depends largely on the positive functional changes in the face of fatigue, and this can only be achieved by regulating the relationship between codifying the training load of effectiveness with the regulation of the nutritional aspect and providing the athlete with all the requirements that work to support energy systems and energy source.

\section{Study Aim:}

The study aims to identify the effect of using a nutritional supplement on some physiological variables and to slow the appearance of fatigue for volleyball players via the following duties:

1) Identifying the effect of using nutritional supplement on some physiological variables on volleyball players.

2) Identifying the effect of the nutritional supplement on slowing the appearance of fatigue among volleyball players. 


\section{Research Method:}

The researcher used the experimental method for one group via using the pre and post measurement design as this is appropriate to the nature of this research.

\section{Research Sample:}

The sample was chosen via purposive way from 36 volleyball players, ranging from 18 to 26 years old.

- Pre and post measurement form for physiological variables (heart rate - lactic acid - vital capacity)

- "nutritional Supplement" Arginine

Chemical Composition: $\mathrm{C}_{6} \mathrm{H}_{14} \mathrm{~N}_{4} \mathrm{O}_{2}$

Weight: $174.204 \mathrm{~g} / \mathrm{mol}$

Free of (fat - sodium - cholesterol - sugars)

Each (1) gram of Arginine

10 Kcal

- Designing the program using the nutritional supplement

The program was designed to use the nutritional supplement (Arginine) on a quantity of yogurt, after reviewing some previous research and studies as well as some scientific journals in this field, and is as follows:

1) The duration of the program for players (3 mønths).

2) The number of weekly doses is three (take a nutritional supplement).

3) After studying previous scientific references and studies, the researcher determines the time during which the players will take the nutritional supplement (15 minutes before the exercise)

The number of training units during the program is (36) training units.

After completing the pre measurements of the research variables, the researcher applied the program on the research sample, and they were (36) volleyball 
players over a period of three months from 1/7/2019 to 1/10/2019 at the rate of three training units per week (Saturday- Monday- Wednesday) as the samples begin to take the nutritional supplement 15 minutes before training and that after eating breakfast about two hours.

Post measurements were taken after the completion of the program in the period from (1/10/2019) to (3/10/2019), as all the measurements of the research were taken for all members of the sample in the same way and in the same order, then the same devices and instruments that were measured for all members of the sample, were used.

\section{View and discuss the results:}

First: Significant differences in lactic acid test

\begin{tabular}{|c|c|c|c|c|}
\hline & LR1 & LR2 & DLR & DDLR \\
\hline Mean & 3.689167 & 2.933333 & -0.755833 & -0.205103 \\
\hline Median & 3.700000 & 2.900000 & -0.750000 & -0.195652 \\
\hline Maximum & 4.600000 & 3.800000 & -0.200000 & -0.068966 \\
\hline Minimum & 2.200000 & 1.600000 & -1.300000 & -0.351351 \\
\hline Std. Dev. & 0.568252 & 0.536656 & 0.309741 & 0.082193 \\
\hline Skewness & -0.293747 & -0.388338 & -0.051331 & -0.159039 \\
\hline Kurtosis & 2.759208 & 2.622213 & 2.120779 & 1.962107 \\
\hline Jarque-Bera & 0.604696 & 1.118926 & 1.175353 & 1.767593 \\
\hline Probability & 0.739081 & 0.571516 & 0.555617 & 0.413211 \\
\hline Sum & 132.8100 & 105.6000 & -27.21000 & -7.383724 \\
\hline Sum Sq. Dev. & 11.30187 & 10.08000 & 3.357875 & 0.236447 \\
\hline Observations & 36 & 36 & 36 & 36 \\
\hline
\end{tabular}


Second: Significant differences in lactic acid in the effort

\begin{tabular}{|l|c|c|c|c|}
\hline & LE1 & LE2 & DLE & DDLE \\
\hline Mean & 7.706111 & 6.586111 & -1.120000 & -0.145992 \\
\hline Median & 7.600000 & 6.650000 & -1.050000 & -0.138079 \\
\hline Maximum & 8.600000 & 7.800000 & -0.400000 & -0.053333 \\
\hline Minimum & 6.400000 & 5.100000 & -2.000000 & -0.253165 \\
\hline Std. Dev. & 0.550918 & 0.701082 & 0.460304 & 0.060135 \\
\hline Skewness & -0.305816 & -0.395735 & -0.289789 & -0.164927 \\
\hline Kurtosis & 2.396107 & 2.325926 & 2.023718 & 1.658147 \\
\hline Jarque-Bera & 1.108170 & 1.621202 & 1.933556 & 2.864059 \\
\hline Probability & 0.574598 & 0.444591 & 0.380306 & 0.238824 \\
\hline Sum & 277.4200 & 237.1000 & -40.32000 & -5.255715 \\
\hline Sum Sq. Dev. & 10.62286 & 17.20306 & 7.415800 & 0.126567 \\
\hline Observations & 36 & 36 & 36 & 36 \\
\hline
\end{tabular}

Third: Significant differences in lactic acid in vital capacity

\begin{tabular}{|l|c|c|c|c|}
\hline & V1 & V2 & DV & DDV \\
\hline Mean & 2809.861 & 3125.056 & 315.1944 & 0.114238 \\
\hline Median & 2755.000 & 3075.000 & 309.0000 & 0.113854 \\
\hline Maximum & 3380.000 & 3590.000 & 580.0000 & 0.238683 \\
\hline Minimum & 2430.000 & 2690.000 & 40.00000 & 0.015094 \\
\hline Std. Dev. & 237.0378 & 217.5479 & 108.2373 & 0.044953 \\
\hline Skewness & 0.402895 & 0.257180 & -0.008211 & 0.465537 \\
\hline Kurtosis & 2.613632 & 2.461989 & 3.257225 & 3.663723 \\
\hline Jarque-Bera & 1.197866 & 0.831034 & 0.099652 & 1.961141 \\
\hline Probability & 0.549397 & 0.659999 & 0.951395 & 0.375097 \\
\hline Sum & 101155.0 & 112502.0 & 11347.00 & 4.112579 \\
\hline Sum Sq. Dev. & 1966542. & 1656448. & 410035.6 & 0.070726 \\
\hline Observations & 36 & 36 & 36 & 36 \\
\hline
\end{tabular}

Second: Discussing the Results:

By using the statistical analysis, appropriate for the nature of this study, it becomes clear from Table (1), (2), (3) that an improvement has occurred in the averages of the measurements of the study variables before and after eating the food supplement to the proposed program that included the first stage of taking the nutritional supplement (Arginine). This is consistent with the results in the (heart rate during rest) as the value of (percentage change rate) is between $(5.19 \%$ to $15.38 \%)$ and the value of (percentage change rate) is between ( $0.63 \%$ to $23.57 \%)$. These values indicate that there are percentage 
differences signifying the improvement of the sample results in in testing the heart rate after eating the nutritional supplement, as the value of (percentage change rate) reached between $(-35.14 \%$ to $-6.90 \%)$. These values indicate that there are percentage differences signifying the improvement of the results sample in testing the level of Lactic Acid after eating the nutritional supplement, as the value of (percentage change rate) reached between $(-25.32 \%$ to $-5.33 \%)$, and these values indicate the presence of percentage differences signifying the improvement of the sample results in testing the level of Lactic Acid after eating the nutritional supplement, as the value of (percentage change rate) is between $(4.23 \%$ to $13.10 \%)$. These values indicate that there are percentage differences signifying the improvement of the results sample in maximum oxygen consumption test after consuming the nutritional supplement, as the value of (percentage change rate) reached between $(1.51 \%$ to $23.87 \%)$. These values indicate that there are percentage differences signifying the improvement of the sample results in testing the vital capacity after eating the nutritional supplement. These values indicate that there are percentage differences signifying the improvement of the sample results after eating the nutritional supplement.

The researcher thinks that these differences in the results are due to the use of the nutritional supplement (Arginine), which works directly to slow the appearance of fatigue in athletes, as well as to the dilatation of blood vessels that allow the passage of a greater amount of things that the muscles need of glucose, water and proteins to continue the performance continuously and the ability of muscles to get rid of harmful waste that hinder its work as quickly as possible, such as Lactic Acid, which in turn, is an amino acid that helps to deliver hormones and in chemical reactions and turns into nitric oxide, which improves the efficiency of chemical reactions that occur within the body and acts as a catalyst for doing physical activity in a large degree. Arginine also helps in working Coenzymes and Enzymes that produce energy and continue the performance continuously, as well as in the urea cycle inside the body as a catalyst, and also in the lactic acid cycle to get rid of harmful waste in the body. All of that had a clear effect on the improvement of differences in all measurements. (Wael Youssef: 94). 
Ahmed Al-Tantawi (2005) believes that the human being represents one biological unit that affects each other and is affected by external stimulus, so some physiological variables and what happens as a result of physical exertion must be studied, including sportive training that causes different biological, functional, and structural changes that include almost all the body's systems. Whenever these changes are positive in order to achieve the functional adaptation required to meet kinetic requirements of the nature of the athlete and the non-athlete, whether during rest or during training, and it leads to achieve progress in the level of performance with high efficiency, economize in consumed energy and adapt for the body systems to perform physical loads with the cooperation of many different systems and organs in the human body.

As well, the reduction of enzymes level is one of the important factors to avoid damage and negative effects on cells due to high acidity resulting from the continued execution of different intensity physical loads as (Martin and Koi) (1997) stated that the enzymatic concentration decreases under the influence of high acidity level, and then mechanics and insurance to avoid destroying the cell (47:81).

Blood is an essential component in the formation of the body's internal structure and is responsible for providing the appropriate internal environment for the body tissues thanks to the exchange process that takes place between it and the fluid between the tissues so that the cells remain in a relatively stable chemical medium. The blood performs many important vital functions and the nature of its composition and its distinctive characteristics help to carry out these functions. The process of its perfusion from one place to another in the body helps to carry out the role of perfusion and transport between the different body cells despite the distances between them and each component of the blood has a specific function, all are completed in the general functions of blood (40:41).

Mohammed Nasr El-Din Radwan (1998) added that a fourth classification can be added under the name: Mixed anaerobic capacity, which indicates the maximum effort the individual exerts for a relatively long period of time, but with a level of intensity and speed that is less than the long anaerobic capacity, so that the performance takes $60-90$ seconds 
as a minimum and up to 2-3 minutes as a maximum as the performance depends on (lactic system) as a source of energy production. (57: 113).

Wael Ramadan (1997) stated that the best way to improve this system is to use activities that last for a performance period ranging from 45 to 60 seconds with high intensity and long rest periods that lead to increased production of lactic acid in the blood as well as an improvement in the athlete's ability to endure a higher level of Lactic acid. As well, the energy characteristics of the lactic acid system are determined as follows (this system does not need oxygen -it depends only on carbohydrates as a source of energy (glucose glycogen). This system produces enough energy to restore a small amount of Adenosine Triphosphate- lactic acid accumulates in the muscles and this is a cause of muscle fatigue $(68: 165)$.

Based on what the results showed about the characteristics and functional changes, the researcher believed that the changes in the functional characteristics of the sample under study are attributed to the body's attempt to create a state of internal balance in response to the physical effort that the player performs, as the continued exposure to pressures of the training load leads to responses and reactions that make an impact on the functional system and they are gathered after each influential load and formed in the end after each repetition of these pressures to a higher level of the functional status with regard to the targeted vital devices. From the foregoing, we find that the research hypotheses have been verified via statistical methods through which the researcher knows the role of the nutritional supplement (Arginine) and its great role in getting rid of Lactic Acid and its vital course in slowing the appearance of muscle fatigue.

Via this presentation and results discussion, the validity of the hypotheses has been proved (there are statistically function differences between the pre and post measurements of the effect of the nutritional supplement on volleyball players in Kafrelsheikh Governorate there are statistically function differences between the pre and post measurements of the effect of the nutritional supplement on the functional efficiency of volleyball players in Kafrelsheikh Governorate - there are statistically function differences between the pre and post measurements of the effect of the nutritional supplement on slowing the appearance of fatigue among volleyball players in Kafrelsheikh Governorate via the presence of 
improvement rates between the pre and post measurements in favor of the post measurement of the effect of taking the nutritional supplement (Arginine) on the sample under study.

The conclusion

Within the research field and its aim, the limits of the used hypotheses and methodology, the reference framework of theoretical and practical studies, and the nature of the sample, via the statistical analysis of the data, the following conclusions have been reached:

1) Eating Arginine Acid as a nutritional supplement before the effort of volleyball player's teams has led to an improvement in the disposal of lactic acid and a decrease in triglycerides in the body.

2) Arginine intake helps in the function of Coenzyme and Enzyme to produce energy and to perform continuously, as well as in the urea cycle inside the body as a catalyst, and in the lactic acid cycle to get rid of harmful waste in the body.

3) The best time to take Arginine Acid 15 minutes before exercise.

4) Eating Arginine Acid as a nutritional supplement before the effort has a significant and important role in continuing the physical performance in a distinct way while slowing the appearance of muscle fatigue.

5) Taking Arginine Acid as a nutritional supplement before the effort helps to increase the efficiency of enzymes in stimulating the biochemical processes of producing the energy needed to continue the performance.

\section{Recommendations:}

In light of the conclusions that were based on the nature of the research. Procedures, goals, statistical treatments and the results, the following recommendations were reached:

1) The necessity of using the proposed program and its results by trainers and workers in the field of sportive training, especially the use of Arginine Acid as 
a nutritional supplement to benefit from it as an aid in various training programs because of its positive impact on some physical capabilities.

2) The importance of taking Arginine Acid as a nutritional supplement to develop some physical capabilities due to its close association with the level of physical performance and slowing the appearance of fatigue in different age stages.

3) The use of Arginine Acid as a nutritional supplement on similar samples of athletes and non-athletes in multiple activities and other age stages so that criteria can be established to be used as a scientific basis in developing sportive programs.

\section{References:}

First: Arabic references:

Effectiveness of developing aerobic and anaerobic ability on some components and their relations to the level of skill performances of basketball junior players, unpublished Ph.D. thesis, Faculty of Physical Education for Boys, Zagazig University.

Ahmed Mohammed Tantawi (2005)

The effect of caffeine associated with competition load in some biochemical variables, the ride and running speed for youth football players, Journal of Physical Education Sciences, Volume 8, number 5

Ameen Abed Allah Khazaal (2015)

Methods of measuring physical exertion in sports, 1st floor, Publishing Al-Kitab Center, Cairo.

Mohammed Nasr Radwan (1998)

The effect of some nutritional factors on slowing the appearance of fatigue and the speed of recovery for athletes, unpublished Ph.D. thesis, Faculty of Physical Education for Boys, Helwan University.

Mohi El-Deen Mahmoud Hassan (1991) 
An open program to develop speed endurance and its impact on the anaerobic threshold and the level of digital achievement for competitors of $800 \mathrm{~m} / \mathrm{g}$, unpublished Master' Degrees Thesis, Faculty of Physical Education for Boys in Cairo, Helwan University.

Wael Mohammed Ramadan (1997)

The effect of taking arginine as a nutritional supplement on slowing the appearance of fatigue for competitors of modern pentathlon, unpublished Ph.D. thesis, Faculty of Physical Education for Boys, Alexandria University.

Wael Youssef Ahmed (2017)

\section{Second: English references}

Exercise Training and Neutrophil function exercise immunology review3096-166,1997

Smith,J.A and Pyne, D.B 